\title{
Spectral transverse instabilities and soliton dynamics in the higher-order multidimensional nonlinear Schrödinger equation
}

\author{
Justin T. Cole, Ziad H. Musslimani \\ Department of Mathematics, Florida State University, Tallahassee, Florida 32306-4510, USA
}

\begin{abstract}
Spectral transverse instabilities of one-dimensional solitary wave solutions to the two-dimensional nonlinear Schrödinger (NLS) equation with fourth-order dispersion/diffraction subject to higher-dimensional perturbations are studied. A linear boundary value problem governing the evolution of the transverse perturbations is derived. The eigenvalues of the perturbations are numerically computed using Fourier and finite difference differentiation matrices. It is found that for both signs of the higher-order dispersion coefficient there exists a finite band of unstable transverse modes. In the long wavelength limit we derive an asymptotic formula for the perturbation growth rate that agrees well with the numerical findings. Using a variational formulation based on Lagrangian model reduction, an approximate expression for the perturbation eigenvalues is obtained and its validity is compared with both the asymptotic and numerical results. The time dynamics of a one-dimensional soliton stripe in the presence of a transverse perturbation is studied using direct numerical simulations. Numerical nonlinear stability analysis is also addressed.
\end{abstract}

Keywords: higher-order dispersion, line solitons, transverse instability

\section{Introduction}

Solitons, or solitary waves, are self-trapped nonlinear modes that exist in many branches of science such as optics [1, 2, 3, 4, 5, fluid mechanics [6, plasmas 17, ultracold gases 8 , biology and chemistry [9, 10]. Among the most intriguing and physically relevant properties associated with these solitary waves is the development of symmetry-breaking instabilities that often lead to the generation of complex nonlinear coherent structures [11].

Modulational instability (MI) is an important example of a symmetry-breaking instability where a constant (in space) amplitude and time-harmonic solution to the underlying governing equation of motion breaks up due to the exponential growth of small modulated perturbations under the combined effects of dispersion/diffraction and nonlinearity. It was first identified in fluid mechanics 12 and plasma physics [13] and subsequently reported in many other areas of physics [14, 15, particularly in nonlinear optics [16, 17, 18, 19, 20, 21]. Very recently [22, modulational instability of constant amplitude waves for the PT-symmetric NLS equation 23, 24, have also been studied.

Another physically relevant modulational instability process is the so-called transverse instability (TI) (see reviews 25, 26, 27]). Contrary to the "conventional" MI (where the base state is constant in all space dimensions), TI describes the break up of a line soliton (a

Email addresses: jcole@math.fsu.edu (Justin T. Cole), muslimani@math.fsu.edu (Ziad H. Musslimani) two-dimensional nonlinear mode localized in one space dimension and uniform in the other) due to the exponential growth of unstable perturbations in the transverse direction. Mathematically speaking, it was first discovered by Zakharov and Rubenchik [28 for the attractive two-dimensional nonlinear Schrödinger equation. They derived an asymptotic expression for the perturbation eigenvalue valid in the long wavelength limit and found that one-dimensional line soliton solutions are transversally unstable. The long time dynamics of the soliton evolution under transverse perturbations is the formation of a train of two-dimensional localized filaments. A similar situation holds for the two-dimensional repulsive (selfdefocusing) NLS equation: a dark soliton stripe becomes unstable against random transverse perturbations and disintegrates into a sequence of vortices (snake-type instability) [29, 30, 31, 32. Transverse instabilities of vector solitons have also been studied theoretically [33, 34, 35], while experimental observation of TI has been reported in the literature for both the scalar and vector cases [36, 37, 38. We point out that TI has also been studied in other settings [39, most notably for the hyperbolic NLS equation [40, 41].

Most of the mathematical models used to study MI and TI processes are based on nonlinear, dispersive and conservative evolution equations with the nonlinear Schrödinger equation being a prototypical example [6. However, higher-order dispersion/diffraction terms become important in certain special regimes. Specifically, fourth-order dispersion has been demonstrated to play an important 
role in fiber optics 42, 43, rogue waves 44 and quantum gravity models 45. Additionally, modulational instability in NLS-type systems with high-order dispersion have been extensively investigated [46, 47, 48, 49, 50, 51.

In this paper, we study spectral transverse instabilities of one-dimensional localized solitary waves subject to two-dimensional perturbations. The model equation considered is based on the two-dimensional nonlinear Schrödinger equation in the presence of higher-order dispersion/diffraction

$$
i \phi_{t}+\frac{1}{2} \Delta \phi-\beta \Delta^{2} \phi+\gamma|\phi|^{2} \phi=0,
$$

where $\phi$ is a complex-valued envelope (amplitude) function, $\beta$ is a real dispersion/diffraction coefficient, $\gamma= \pm 1$, $\Delta$ is the two-dimensional Laplacian describing dispersion/diffraction in the transverse $(x, y)$ plane and $\Delta^{2}$ is the so-called bi-Laplacian (or biharmonic) operator. When $\beta=0$, Eq. (1) successfully models several physical phenomena related to optics 2, 6, Bose-Einstein condensates [52], and fluid mechanics [53]. For nonzero $\beta$, Eq. (1) can be viewed as a special case of the more general complex Swift-Hohenberg equation derived in [54] as a model of optical wave propagation in a cavity near the onset of lasing. Moreover, when $\beta>0$ Eq. (1) models optical beams whose diameter is on the order of its wavelength (linearly polarized nonparaxial beams), where $t$ plays the role of propagation distance. In this regard, Eq. (1) could be viewed as an "intermediate" case between the paraxial wave and Helmholtz equations [45, 55, 56, 57].

A linear evolution equation for the transverse perturbation is obtained. Using separation of variables, we find an eigenvalue system whose spectrum is numerically computed with the help of differentiation matrices. It is found that when $\beta$ is positive there exists a finite band of unstable transverse modes and the soliton stripe is unstable against perturbations with small wavenumbers. This long wavelength instability seems to disappear when $\beta$ is negative for solitons with small amplitude. In the long wavelength limit we derive an asymptotic formula for the perturbation growth rate that agrees well with the numerical findings. This perturbative result coincides with the formula obtained by Zakharov and Rubenchik [28] for the "classical" two-dimensional nonlinear Schrödinger equation $(\beta=0)$. Based on a variational approach, an approximate expression for the perturbation eigenvalues is also obtained and its validity is compared with the asymptotics as well as the numerical results. The time dynamics of the soliton stripe superimposed with a transverse perturbation is investigated by numerically solving the Cauchy problem associated with Eq. (1). Finally, numerical nonlinear stability results are also presented.

The outline of the paper is as follows. In Sec. 2 we identify families of one-dimensional line soliton solutions followed by (Sec. 3) a thorough analysis of their linear stability. In Secs. 4 and 5 we report on analytical results for the linear stability analysis. Comparison with direct sim- ulations is presented in Sec. 6. We conclude the discussion in Sec. 7

\section{Line (stripe) solitons}

We start the discussion by considering a family of onedimensional soliton solutions to Eq. (1) that are independent of the transverse coordinate $y$

$$
\phi(x, y, t)=\psi(x, \mu) e^{i \mu t}
$$

which satisfy the nonlinear boundary value problem

$$
\frac{1}{2} \partial_{x}^{2} \psi-\beta \partial_{x}^{4} \psi+\gamma|\psi|^{2} \psi=\mu \psi
$$

Equation (3) is supplemented with the boundary conditions: $\psi$ tends to zero sufficiently fast as $|x|$ tends to infinity. The eigenvalue $\mu$ is referred to as the soliton propagation constant and its sign is adjusted depending on the signs of $\beta$ and $\gamma$. We note that Eq. (3) in the presence of an external periodic potential has been studied in [58. To determine the eigenfunction and eigenvalue pair $(\psi, \mu)$ we numerically integrate Eq. (3) using a spectral renormalization method [59] (see details in Appendix A). It is worth mentioning that a special solution for Eq. (3) is known to exist for $\beta>0, \gamma=+1$ and is given by 60

$$
\psi(x)=\sqrt{\frac{3}{40 \beta}} \operatorname{sech}^{2}\left(\frac{x}{\sqrt{40 \beta}}\right), \quad \mu=\frac{1}{25 \beta} .
$$

In Figs. A.1 and A.2 we show typical examples of nonlinear mode profiles (solutions of Eq. (3) for both positive and negative values of $\beta, \gamma$ and $\mu$. First, we address the positive $\beta$ case of which three different solutions corresponding to different soliton eigenvalues $\mu$ are depicted in Fig. A.1. It is seen that the soliton amplitude becomes larger as $\mu$ increases, and as a result the soliton profile becomes more localized. The soliton shown in Fig. A.1(a) corresponds to analytical solution (4) for $\beta=1 / 10$. It is interesting to note that the soliton shapes given in Figs. A.1(b) and A.1(c) display a weak oscillatory tail and in some space domains become negative. This is contrary to the analytical solution in Eq. (4) which is postivedefinite and monotonically decaying as $x$ approaches $\pm \infty$. We can understand this oscillatory feature by examining the large $x$ asymptotics of Eq. (3) given by

$$
\frac{1}{2} \partial_{x}^{2} \psi-\beta \partial_{x}^{4} \psi \approx \mu \psi
$$

Making the ansatz $\psi(x) \sim \exp (s x)$ we find nontrivial solutions for values of the exponent $s$ satisfying

$$
s= \pm\left(\frac{1}{4 \beta}(1 \pm \sqrt{1-16 \beta \mu})\right)^{1 / 2} .
$$

It is evident that the exponent $s$ is real when $\beta=1 / 10$ and $\mu=2 / 5$, whereas it becomes complex for $\mu=2$ and 4 . This explains the oscillatory behavior of the soliton tails. 
Next, consider the negative $\beta$ case which appears to be profoundly different. As noted in 61 and 62 there are no localized solutions to Eq. (3) for positive $\gamma$ and $\mu$. Thus we limit ourselves to the case where $\gamma=-1$ and negative $\mu$ values. With this in mind, we show in Fig. A.2 typical solutions for various soliton eigenvalues. As one notices, the soliton profiles show a drastic difference in comparison to their $\beta>0$ counterparts in the sense that they become highly oscillatory. In the asymptotic limit (6), we also observe that no exponentially decaying solutions exist when

$$
1>16 \beta \mu>0, \quad \beta, \mu<0,
$$

since $\operatorname{Re}(s)=0$. For a fixed transverse power $N=$ $\int_{\mathbb{R}}|\psi|^{2} d x$, the soliton existence curves are summarized in a $(\beta, \mu)$ phase diagram (see Fig. A.3). As $\beta \rightarrow 0^{+}$, the eigenvalue approaches the classic NLS propagation constant $\mu=N^{2} / 8$, while as $\beta \rightarrow 0^{-}$numerical simulations show $\mu \rightarrow-\infty$. When $\beta<0$, localized solutions are found for eigenvalues below the curve $\mu=1 /(16 \beta)$, while in region (7) modes with exponential decay do not exist. As $|\beta| \rightarrow \infty$ the soliton solutions develop slower exponential decay and vice vera as $|\beta| \rightarrow 0$. Typical soliton solutions for large $\beta$ values are shown in Fig. A.4 for a fixed power.

Having found nonlinear modes for various sets of model parameters, we next turn our attention to the question of linear stability analysis.

\section{Linear stability analysis}

To study the linear stability of the line soliton solutions found above, we write a weakly perturbed solution of Eq. (1) in the form

$$
\phi(x, y, t)=[\psi(x)+\varepsilon \eta(x, y, t)] e^{i \mu t},
$$

where $|\varepsilon| \ll 1$ is a dimensionless parameter used to measure the strength of the perturbation and $\eta$ is a complex transverse perturbation that depends on $x, y$ and $t$. Note that we are considering here the linear stability of a realvalued wave function $\psi$. Substituting the ansatz (8) into Eq. (1) we find to order $\varepsilon$

$$
i \eta_{t}-\mu \eta+\frac{1}{2} \Delta \eta-\beta \Delta^{2} \eta+\gamma \psi^{2}\left(2 \eta+\eta^{*}\right)=0 .
$$

Equation is a linear constant coefficient in $y$ and $t$ (and variable in $x$ ) PDE that governs the evolution of the perturbation $\eta$ subject to the following boundary conditions: $\eta \rightarrow 0$ sufficiently fast as $x^{2}+y^{2} \rightarrow \infty$. With this in mind, we decompose the perturbation in terms of its Fourier modes and assume that the $t$-evolution is exponential

$\eta(x, y, t)=\int_{\mathbb{R}}\left(f_{q}(x) e^{i(q y+\omega(q) t)}+g_{q}^{*}(x) e^{-i\left(q y+\omega^{*}(q) t\right)}\right) d q$

where $f_{q}, g_{q}$ are the perturbation Fourier modes assumed to be localized in $x$ and $q$ with corresponding wavenumber $q$ and complex frequency $\omega(q)$ that measures the perturbation growth rate. Substituting anstatz 10 into Eq. (9) and collecting terms proportional to $\exp (i(q y+\omega t))$ and $\exp \left(-i\left(q y+\omega^{*} t\right)\right)$ independently results in the following non-Hermitian eigenvalue system

$$
\left(\begin{array}{cc}
0 & \mathbb{M}_{12} \\
\mathbb{M}_{21} & 0
\end{array}\right)\left(\begin{array}{l}
F_{q} \\
G_{q}
\end{array}\right)=\omega\left(\begin{array}{c}
F_{q} \\
G_{q}
\end{array}\right)
$$

where, by definition, $F_{q}=f_{q}+g_{q}, G_{q}=f_{q}-g_{q}$ and the off-diagonal elements are

$$
\begin{aligned}
& \mathbb{M}_{21}=\mathbb{L}_{1}-q^{2} / 2-\beta q^{4}+2 \beta q^{2} \partial_{x}^{2}, \\
& \mathbb{M}_{12}=\mathbb{L}_{2}-q^{2} / 2-\beta q^{4}+2 \beta q^{2} \partial_{x}^{2},
\end{aligned}
$$

for

$$
\begin{aligned}
& \mathbb{L}_{1}=-\mu+\partial_{x}^{2} / 2-\beta \partial_{x}^{4}+3 \gamma \psi^{2} \\
& \mathbb{L}_{2}=-\mu+\partial_{x}^{2} / 2-\beta \partial_{x}^{4}+\gamma \psi^{2}
\end{aligned}
$$

Note that the linear operators $\mathbb{L}_{1}$ and $\mathbb{L}_{2}$ are self-adjoint with respect to the standard $L^{2}(\mathbb{R})$ real-valued inner product

$$
\left\langle u_{1}, u_{2}\right\rangle_{L^{2}(\mathbb{R})}=\int_{\mathbb{R}} u_{1} u_{2} d x,
$$

which induces the norm

$$
\|u\|_{2}=\left(\int_{\mathbb{R}} u^{2}(x) d x\right)^{1 / 2} .
$$

It is obvious from Eq. 10 that for a fixed wavenumber $q$, the eigenvalue $\omega=\omega(q)$ with nonzero imaginary component will grow exponentially in $t$, hence the perturbation $\eta$ becomes unbounded. In this case, we say that the nonlinear mode $\phi(x, y, t)=\psi(x, \mu) \exp (i \mu t)$ is linearly unstable to transversally modulated perturbations. To determine if such modes are indeed linearly stable or not we solve eigenvalue problem (11) and compute the spectrum $\omega$ as a function of the transverse wavenumber $q$. This is accomplished by numerically approximating the second and fourth-order derivatives $\partial_{x}^{2}$ and $\partial_{x}^{4}$ using, for example, spectral or finite difference differentiation matrices on a large computational domain 63]. The imaginary part of the eigenvalue $\omega(q)$ (corresponding to TI) is shown in Fig. A.5 (a) for $\beta=1 / 10, \gamma=+1$ and various values of the transverse wavenumber $q$. The solid, dashed and dasheddotted curves correspond to different soliton eigenvalues $\mu$ whose shapes are depicted in Fig. A.1. The numerical results reveal the existence of a finite band $\mathcal{I}=\left(0, q_{\text {cut }}(\mu, \beta)\right)$ of Fourier modes that grow exponentially in $t$ and force the soliton to disintegrate (see Sec. 6 for detailed numerical simulations). The numerical findings suggest that the measure of the interval $\mathcal{I}$ is a monotonic function of $\mu$. Importantly, the instability appears to develope for any small wavenumber $q$ and attains its maximum value $\operatorname{Im} \omega_{\max } \equiv \max _{q} \operatorname{Im}(\omega(q))$ at some wavenumber $q_{\max }$. We note that $q$, large soliton amplitudes experience the 
fastest instability development. The linear stability analysis suggests that the line soliton is stable against short wavelength transverse perturbations (see Sec. 6).

In order to understand the role of the biharmonic dispersion coefficient $\beta$ in the development of TI we have repeated the above numerical experiments for moderate values of $\beta$. The main characteristics of the instability pattern remain unchanged with the slight exception that the unstable band measure shrinks and the maximum unstable eigenvalue decreases (see Fig. A.5(b)). Hence a sizable instability pattern is observed on longer $t$ scales. As an example, for fixed $\mu=4, \gamma=+1$ we find for $\beta=0.1$ that $q_{\text {cut }} \approx 2.65$ and $\operatorname{Im} \omega_{\max } \approx 5.04$ at $q_{\max } \approx 2.06$. Compare this with $\beta=0.25$ where $q_{\text {cut }} \approx 2.17$ and $\operatorname{Im} \omega_{\max } \approx 4.87$ is located at $q_{\max } \approx 1.71$. This result is somehow not so surprising since for positive $\beta$ the operator $\Delta-\beta \Delta^{2}$ produces larger effective dispersion/diffraction than the Laplacian alone, hence it tends to weaken, but not eliminate, the instability.

Next we proceed with the negative $\beta$ case. As before, system (11) is solved numerically for the perturbation eigenvalues $\omega(q)$ with negative values of $\gamma$ and $\mu$. In sharp contrast to what we have so far observed, in Fig. A.6(a) the unstable linear spectrum $\operatorname{Im} \omega(q)$ with relatively small soliton eigenvalues $|\mu|$ are now compactly supported on the interval $\mathcal{I}_{q}=\left(q_{\text {cut }}^{(1)}(\beta, \mu), q_{\text {cut }}^{(2)}(\beta, \mu)\right)$. Moreover, the linear stability analysis predicts that long wavelength transverse perturbations do not grow exponentially in $t$. The length of this instability island $\mathcal{I}_{q}$ expands with increasing soliton eigenvalue $|\mu|$. The numerical results seem to suggest that higher amplitude solitons are more susceptible to instability against long wavelength perturbations. For fixed nonlinearity coefficient $\gamma$ and soliton eigenvalue $\mu$, the moderate value of $\beta=0.25$ seems to have little effect on the overall nature of the spectrum, as one sees in Fig. A.6(b).

In the large $\beta$ limit, higher-order dispersion/diffraction is dominant and leads to a sizable suppression of transverse instability. In Fig. A.7 we show typical examples of the unstable eigenvalues for the soliton solutions shown in Fig. A.4 corresponding to various values of $\beta$. For both signs of the biharmonic coefficient, increasing its magnitude reduces the interval of unstable wavenumbers for which $\operatorname{Im}(\omega) \neq 0$.

\section{Asymptotic analysis: long wavelength limit}

To support our numerical findings and to gain further insight into the instability development we resort in this section to perturbation theory and derive an asymptotic formula for the perturbation eigenvalues $\omega(q)$ valid in the long wavelength limit i.e. $q \rightarrow 0$. We start by expanding the perturbation eigenfunctions $F_{q}$ and $G_{q}$ as well as the eigenvalues $\omega(q)$ in an asymptotic series for small $q$ :

$$
\begin{gathered}
\omega=q \omega_{1}+q^{2} \omega_{2}+\cdots \\
F_{q}(x)=F_{0}(x)+q F_{1}(x)+q^{2} F_{2}(x)+\cdots
\end{gathered}
$$

$$
G_{q}(x)=G_{0}(x)+q G_{1}(x)+q^{2} G_{2}(x)+\cdots
$$

Substituting expansions (18)-20 into boundary value problem (11) and collecting terms at each order of $q$ we find

$$
\begin{array}{cll}
O(1): & \mathbb{L}_{1} F_{0}=0, & \mathbb{L}_{2} G_{0}=0, \\
O(q): & \mathbb{L}_{1} F_{1}=\mathcal{P}_{1}^{F}, & \mathbb{L}_{2} G_{1}=\mathcal{P}_{1}^{G}, \\
O\left(q^{2}\right): & \mathbb{L}_{1} F_{2}=\mathcal{P}_{2}^{F}, & \mathbb{L}_{2} G_{2}=\mathcal{P}_{2}^{G},
\end{array}
$$

and in general for any order $n \geq 3$

$$
O\left(q^{n}\right): \quad \mathbb{L}_{1} F_{n}=\mathcal{P}_{n}^{F}, \quad \mathbb{L}_{2} G_{n}=\mathcal{P}_{n}^{G},
$$

where the functions $\mathcal{P}_{n}^{F}$ and $\mathcal{P}_{n}^{G}$ depend on the previous $F_{l}$ and $G_{l}$ for $l=0, \ldots, n-1$. All the eigenfunctions $F_{n}$ and $G_{n}$ are assumed to be smooth and belong to the space of square integrable functions defined on the whole real line. Our aim is to solve Eqns. (21)-23) successively. Since Eq. (1) admits gauge and space-translation invariance symmetries it follows

$$
\mathbb{L}_{1}\left(\partial_{x} \psi\right)=0, \quad \mathbb{L}_{2}(\psi)=0 .
$$

Relation 25 combined with the asymptotic result in Eq. (6) imply that the dimension of the kernel (in $L^{2}(\mathbb{R})$ ) of $\mathbb{L}_{j}, j=1,2$ is at most two. We have numerically solved the eigenvalue problems $\mathbb{L}_{1} v_{1}=\lambda_{1} v_{1}$ and $\mathbb{L}_{2} v_{2}=$ $\lambda_{2} v_{2}$ by representing each derivative by its corresponding finite-difference or spectral differentiation matrix with zero Dirichlet boundary conditions on a sufficiently large spatial domain and collocating the function $\psi^{2}(x)$ along the matrix diagonal. Our numerical findings strongly indicate that the only eigenfunctions of $\mathbb{L}_{1}$ and $\mathbb{L}_{2}$ that belong to the $L^{2}(\mathbb{R})$ space, with corresponding zero eigenvalues, are precisely those functions satisfying 25). With this at hand, we shall therefore assume throughout the rest of this paper that there exists $\left\{\beta_{0}, \mu_{0}, \gamma_{0}\right\}$ for which the dimension of the kernel of $\mathbb{L}_{j}, j=1,2$ is exactly one. Under the above assumption, we write the homogenous solution to Eq. 21) as $F_{0}=C_{1} \partial_{x} \psi$ and $G_{0}=C_{2} \psi$ for nonzero constants $C_{1}$ and $C_{2}$. At order $q$, we have $\mathcal{P}_{1}^{F}=\omega_{1} G_{0}$ and $\mathcal{P}_{1}^{G}=\omega_{1} F_{0}$. Differentiating Eq. 3. with respect to $\mu$ we find $F_{1}=C_{2} \omega_{1} \partial_{\mu} \psi$. By the Fredhlom Alternative theorem, an $L^{2}(\mathbb{R})$ solution to $\mathbb{L}_{2} G_{1}=\mathcal{P}_{1}^{G}$ exists and is given by $G_{1}=C_{1} \tilde{\psi}$ such that $\mathbb{L}_{2} \tilde{\psi}=\omega_{1} \partial_{x} \psi$ (up to a homogenous solution). At order $q^{2}$, we have $\mathcal{P}_{2}^{F}=C_{1} \omega_{1} \tilde{\psi}+C_{2} \omega_{2} \psi+C_{1} \partial_{x} \psi / 2-2 \beta C_{1} \partial_{x}^{2}\left(\partial_{x} \psi\right)$ and $\mathcal{P}_{2}^{G}=C_{2} \omega_{1}^{2} \partial_{\mu} \psi+C_{1} \omega_{2} \partial_{x} \psi+C_{2} \psi / 2-2 \beta C_{2} \partial_{x}^{2} \psi$. The solvability condition

$$
\left\langle\psi, \mathcal{P}_{2}^{G}\right\rangle_{L^{2}(\mathbb{R})}=0
$$

gives the following expression for the perturbation spectrum

$$
\omega= \pm i q \Omega^{1 / 2}+O\left(q^{2}\right),
$$


where

$$
\Omega=\frac{4 \beta\left\|\partial_{x} \psi\right\|_{2}^{2}+\|\psi\|_{2}^{2}}{\partial_{\mu}\|\psi\|_{2}^{2}} .
$$

Thus for positive $\beta$ the numerator in Eq. 28 is positivedefinite, hence all solitary wave solutions to Eq. (3) satisfying the "slope" condition

$$
\partial_{\mu}\|\psi\|_{2}^{2}>0
$$

are unstable against transverse perturbations with large wavelength and as a result grow exponentially in $t$. On the other hand, for negative $\beta$ all transverse perturbations with wavenumbers satisfying $|q| \ll 1$ remain bounded for short $t$ scales if $\|\psi\|_{2}^{2}>-4 \beta\left\|\partial_{x} \psi\right\|_{2}^{2}$ and $\partial_{\mu}\|\psi\|_{2}^{2}<0$. hence this family of solutions are always unstable to perturbations with long transverse wavelength. To apply criterion 27) for any numerically generated stripe soliton, it suffices to compute the power $\|\psi\|_{2}^{2}$ and the "kinetic energy" term $4|\beta|\left\|\partial_{x} \psi\right\|_{2}^{2}$ curves as a function of the soliton propagation constant $\mu$ for various fixed values of $\beta$. It is clear from Fig. A.8, that for positive $\beta$, the soliton power is an increasing function of $\mu$, which combined with the slope condition 29 yields transverse instability. On the other hand, when $\beta<0$ the slope condition alone is insufficient and one must also examine the behavior of the numerator appearing in Eq. (28). To do so, we show in Fig. A.8 the soliton power (solid line, see also Fig. A.9) as well as the quantity $4|\beta|\left\|\partial_{x} \psi\right\|_{2}^{2}$ (dashed line) as a function of $\mu$ for several negative $\beta$ values. Contrary to the previous case, the soliton power curves are now a decreasing function of the soliton eigenvalue, hence $\partial_{\mu}\|\psi\|_{2}^{2}<0$. Moreover, the two quantitates $4 \beta\left\|\partial_{x} \psi\right\|_{2}^{2},\|\psi\|_{2}^{2}$ intersect each other at some $\mu=\mu^{*}(\beta)$, giving rise to transverse instability for $\mu<\mu^{*}$.

To confirm result (27) we have compared it with the numerically computed TI eigenvalues for $\beta=1 / 10, \gamma=+1$ and various soliton eigenvalues. The quantity $\partial_{\mu}\|\psi\|_{2}^{2}$ is computed using a centered finite difference stencil. A summary of the findings are shown in Fig. A.10. For small wavenumbers q, Eq. 27) agrees well with the numerically generated perturbation eigenvalues (see Table 1 in Sec. 5]. Similar tests have been performed for negative $\beta$ values. In particular, for small soliton eigenvalues $|\mu|$ we find that the term $4|\beta|\left\|\partial_{x} \psi\right\|_{2}^{2}$ is smaller than $\|\psi\|_{2}^{2}$ and $\partial_{\mu}\|\psi\|_{2}^{2}<0$ (see Fig. A.8, hence $\omega$ is real and the soliton is linearly stable (see Fig. A.11(a)). Moreover, as $|\mu|$ increases $4|\beta|\left\|\partial_{x} \psi\right\|_{2}^{2}$ grows larger than $\|\psi\|_{2}^{2}$ and the perturbation eigenvalues become purely complex (linearly unstable) for small $q$ (see Figs. A.11(b) and A.11(c)).

\section{Variational formulation}

The previous approaches to compute the transverse dispersion relation $\omega(q)$ were based on numerical integration of boundary eigenvalue problem (11) as well as on the asymptotic analysis valid for small wavenumbers. In this section we take a different approach to compute and study the development of the spectral instability using a variational formulation. The central idea behind the method is to reformulate Eq. (1) in terms of its corresponding Lagrangian functional, then make a suitable solution ansatz that depends on few degrees of freedom and obtain an effective Lagrangian by integrating over a reduced number of degrees of freedom. By taking the variational derivative of the reduced Lagrangian, we obtain a coupled set of PDEs that are later used to study perturbation theory. We remark that this variational approach has been used to obtain approximate analytical forms for solitons in various settings 64, 65, 66.

We begin by considering the Lagrangian functional

$$
\mathcal{L}=\frac{1}{2} \iiint_{\mathbb{R}^{2} \times[0, T]} \mathcal{G} d x d y d t
$$

$$
\mathcal{G}=i\left(\phi \partial_{t} \phi^{*}-\phi^{*} \partial_{t} \phi\right)+|\nabla \phi|^{2}+2 \beta|\Delta \phi|^{2}-\gamma|\phi|^{4},
$$

that using the Euler-Lagrange equations with the respect to the solution $\phi(x, y, t)$ reproduces Eq. (1). Our strategy for computing the transverse instability dispersion curves is as follows. First, let $\varphi_{\mu}(x)$ be a smooth and real-valued localized solution to Eq. (3) corresponding to eigenvalue $\mu$. Multiplying that equation by $\varphi_{\mu}(x)$ and integrating over the whole real line gives

$$
\gamma\left\|\varphi_{\mu}\right\|_{4}^{4}=\mu\left\|\varphi_{\mu}\right\|_{2}^{2}+\frac{1}{2}\left\|\partial_{x} \varphi_{\mu}\right\|_{2}^{2}+\beta\left\|\partial_{x}^{2} \varphi_{\mu}\right\|_{2}^{2}
$$

where we define the $L^{p}(\mathbb{R})$ norm of $\varphi_{\mu}$ by

$$
\left\|\varphi_{\mu}\right\|_{p}=\left(\int_{\mathbb{R}}\left(\varphi_{\mu}\right)^{p} d x\right)^{1 / p}
$$

We now make the ansatz solution to Eq. (1) that is a one-dimensional soliton modulated in the transverse $y$ direction i.e.

$$
\phi^{A}(x, y, t)=A(t, y) \varphi_{\mu}(x) e^{i \mu t}
$$

where $A$ is a complex-valued amplitude that depends on $y$ and $t$. This separation of variables approach, where the trial function $\varphi_{\mu}(x)$ is kept exact, simplifies the calculations and allows one to relate back to the stability problem by choosing the amplitude $A$ to depend on $t$ and $y$ only. Also, in the method proposed here, the solution $\varphi_{\mu}(x)$ is either known analytically, as is the case in Eq. (4), or may be numerically generated. A more general ansatz $\phi^{A}$ that includes more parameters (e.g. soliton width and phase) is possible (see 67] for a classical NLS example). This could lead to better approximations for the perturbation eigenvalue $\omega$, however the analysis could become cumbersome. The nice thing about our ansatz is that it simplifies the calculations, captures the structure of the unstable spectrum and agrees well with the previous approaches. 
Substituting (34) into the Lagrangian (30) and integrating the $x$ degree of freedom we obtain the effective Lagrangian

$$
L_{\mathrm{eff}}\left(A, A^{*}\right)=\left.\frac{1}{2} \int_{\mathbb{R}} \mathcal{G}\left(\phi, \partial_{t} \phi, \nabla \phi, \Delta \phi\right)\right|_{\phi=\phi^{A}} d x,
$$

that after some calculations simplifies to

$$
\begin{aligned}
L_{\mathrm{eff}}\left(A, A^{*}\right) & =\frac{i}{2}\left(A \partial_{t} A^{*}-A^{*} \partial_{t} A\right) E_{1}+\mu|A|^{2} E_{1} \\
& +\frac{1}{2}|A|^{2} E_{2}+\frac{1}{2}\left|\partial_{y} A\right|^{2} E_{1}+\beta|A|^{2} E_{3} \\
& -\beta\left(A^{*} \partial_{y}^{2} A+A \partial_{y}^{2} A^{*}\right) E_{2}+\beta\left|\partial_{y}^{2} A\right|^{2} E_{1} \\
& -\frac{\gamma}{2}|A|^{4} E_{4}
\end{aligned}
$$

where $E_{j}, j=1,2,3,4$ are positive constants that implicitly depend only on $\beta, \gamma$ and $\mu$

$$
\begin{aligned}
& E_{1}=\int_{\mathbb{R}} \varphi_{\mu}^{2} d x, \\
& E_{2}=\int_{\mathbb{R}}\left(\partial_{x} \varphi_{\mu}\right)^{2} d x, \\
& E_{3}=\int_{\mathbb{R}}\left(\partial_{x}^{2} \varphi_{\mu}\right)^{2} d x, \\
& E_{4}=\int_{\mathbb{R}} \varphi_{\mu}^{4} d x .
\end{aligned}
$$

To obtain the dynamical equation that governs the evolution of the amplitude $A$, we use the first variation principle $\delta \mathcal{L}=0$ via the Euler-Lagrange equation

$$
\frac{\partial L_{\mathrm{eff}}}{\partial A^{*}}-\frac{\partial}{\partial t} \frac{\partial L_{\mathrm{eff}}}{\partial\left(\partial_{t} A^{*}\right)}-\frac{\partial}{\partial y} \frac{\partial L_{\mathrm{eff}}}{\partial\left(\partial_{y} A^{*}\right)}+\frac{\partial^{2}}{\partial y^{2}} \frac{\partial L_{\mathrm{eff}}}{\partial\left(\partial_{y}^{2} A^{*}\right)}=0
$$

A straightforward calculation gives

$$
\begin{aligned}
i \partial_{t} A & -\left(\mu+\frac{E_{2}}{2 E_{1}}+\beta \frac{E_{3}}{E_{1}}\right) A+\left(\frac{1}{2}+2 \beta \frac{E_{2}}{E_{1}}\right) \partial_{y}^{2} A \\
& -\beta \partial_{y}^{4} A+\gamma \frac{E_{4}}{E_{1}}|A|^{2} A=0 .
\end{aligned}
$$

With this result at hand, we next proceed with the question of stability. Note that $A=1$ is a fixed-point of Eq. 42 which implies that $\phi^{A}$ is an exact solution to Eq. (3). A small perturbation around the steady and homogenous state in the form

$$
A(t, y)=1+\varepsilon A_{1}(t, y)+\cdots, \quad \varepsilon \ll 1,
$$

can be interpreted as a perturbation of the soliton $\varphi_{\mu}$. Since, in this case, Eq. 34 reads $\phi^{A}(x, y, t)=$ $\left[\varphi_{\mu}(x)+\varepsilon A_{1}(t, y) \varphi_{\mu}(x)+\cdots\right] \exp (i \mu t)$, where $A_{1} \varphi_{\mu}$ can be thought of as transverse perturbation $\eta$. Substituting the asymptotic expansion 43 into Eq. 42 one finds to leading order in $\varepsilon$

$$
\gamma E_{4}=\mu E_{1}+\frac{1}{2} E_{2}+\beta E_{3}
$$

which is identical to Eq. 32. At order $\varepsilon$ we find the following linear evolution equation

$$
i \partial_{t} A_{1}+\left(\frac{1}{2}+\frac{2 \beta E_{2}}{E_{1}}\right) \partial_{y}^{2} A_{1}-\beta \partial_{y}^{4} A_{1}+\frac{\gamma E_{4}}{E_{1}}\left(A_{1}+A_{1}^{*}\right)=0 .
$$

Next we decompose the envelope $A_{1}$ into its Fourier integral representation

$$
A_{1}(t, y)=\int_{\mathbb{R}}\left(u_{q} e^{i(q y+\omega t)}+v_{q}^{*} e^{-i\left(q y+\omega^{*} t\right)}\right) d q,
$$

where $u_{q}$ and $v_{q}$ are the Fourier coefficients assumed to be constant in $t, y$ and localized in $q$. Here $q \in \mathbb{R}$ is the perturbation wavenumber and $\omega$ is (in general) the complex frequency measuring the perturbation growth rate. Upon substituting Eq. 46 into 45 one obtains the nonHermitian eigenvalue problem

$$
\left(\begin{array}{cc}
0 & \mathbb{W}_{12} \\
\mathbb{W}_{21} & 0
\end{array}\right)\left(\begin{array}{c}
U_{q} \\
V_{q}
\end{array}\right)=\omega\left(\begin{array}{c}
U_{q} \\
V_{q}
\end{array}\right)
$$

Here we define $U_{q}=u_{q}-v_{q}, V_{q}=u_{q}+v_{q}$ and

$$
\begin{aligned}
& \mathbb{W}_{12}=\frac{2 \gamma E_{4}}{E_{1}}-\left(\frac{1}{2}+\frac{2 \beta E_{2}}{E_{1}}\right) q^{2}-\beta q^{4} \\
& \mathbb{W}_{21}=-\left(\frac{1}{2}+\frac{2 \beta E_{2}}{E_{1}}\right) q^{2}-\beta q^{4}
\end{aligned}
$$

The advantage of this variational approach over the one presented in Sec. 3 is that eigenvalue system (47) is exactly solvable, unlike the one given in Eq. (11) where only numerical diagonalization and long wavelength perturbation analysis is available. Solving for the eigenvalue $\omega$ gives the dispersion relation

$$
\begin{aligned}
\omega^{2}=q^{2} & {\left[\left(\frac{1}{2}+2 \beta \theta_{1}\right)+\beta q^{2}\right] } \\
& \times\left\{\left(\frac{1}{2}+2 \beta \theta_{1}\right) q^{2}+\beta q^{4}-2 \gamma \theta_{2}\right\},
\end{aligned}
$$

where $\theta_{1} \equiv E_{2} / E_{1}$ and $\theta_{2} \equiv E_{4} / E_{1}$ are positive constants. For the exact solution given in Eq. (4) the dispersion relation simplifies to

$$
\omega_{\text {exact }}^{2}=q^{2}\left(\frac{27}{50}+\beta q^{2}\right)\left[\frac{27}{50} q^{2}+\beta q^{4}-\frac{18}{175 \beta}\right] .
$$

From this equation, it is clear that $\operatorname{Im}\left(\omega_{\text {exact }}\right) \neq 0$ when $\beta q^{2}\left(27 / 50+\beta q^{2}\right)<18 / 175$, i.e. for small $q$. Furthermore, by letting $q_{1}=\frac{1}{2 \beta}+2 \theta_{1}$ and $K^{2}=\frac{2 \gamma \theta_{2}}{\beta}$ for $\beta \neq 0$ Eq. (50) is factored as

$$
\omega^{2}=\beta^{2} q^{2}\left(q^{2}+q_{1}\right)\left(q^{2}-q_{2}^{2}\right)\left(q^{2}+q_{3}^{2}\right) .
$$

Here we define

$$
q_{2}^{2}=\frac{-q_{1}+\sqrt{q_{1}^{2}+4 K^{2}}}{2},
$$




\begin{tabular}{|c|c|c|c|}
\hline$\mu$ & $\Omega^{1 / 2}$ & $\Lambda^{1 / 2}$ & Numerical \\
\hline 0.4 & 0.866 & 0.745 & 0.866 \\
\hline 2 & 1.992 & 1.772 & 1.992 \\
\hline 4 & 2.931 & 2.635 & 2.931 \\
\hline
\end{tabular}

Table 1: Numerical values (rounded to the third decimal digit) of $\Omega^{1 / 2}$ and $\Lambda^{1 / 2}$ obtained from formulas 28 and $\sqrt{55}$ for various soliton propagation constants. For comparison, we also show the numerically computed instability eigenvalues generated from eigenvalue problem 111 for $q=0.01$. Parameters are $\beta=0.1$ and $\gamma=+1$.

with $q_{3}^{2}$ a positive quantity that depends on $q_{1}$ and $K^{2}$. Since $\gamma \beta>0$ (the only case considered here) it follows that $K^{2}$ is positive.

Thus the soliton stripe $\psi$ is transversely unstable if $\omega^{2}<0$ and linearly stable otherwise. When $\beta$ is positive it follows that $q_{1}>0$, in which case $q_{2}^{2}$ remain positive as well. This in turn implies the existence of a finite band of unstable Fourier modes $q \in\left(0, q_{2}\right)$ for which $\operatorname{Im} \omega$ is nonzero. This result is consistent with the numerical and perturbation findings obtained in Secs. 3 and 4 . Figure A.12(a-c) shows the instability growth rate $\operatorname{Im} \omega$ as a function of the unstable modes $q$ for typical parameters $\beta=1 / 10$ and $\gamma=+1$. For comparison, we also show the numerically computed eigenvalues obtained by solving Eq. (11). Note that the variational curve in Fig. A.12(a) is given by the analytic formula (51). As one can see, there is a relatively good qualitative agreement between the variational and numerical approaches. Both methods predict nearly identical unstable band measure, location of the most unstable mode $q_{\max }$, as well as maximum growth rates (with a slight difference from the numerical value Im $\omega_{\max }$ of about $8.7,4.8,3.3 \%$ in Figs. A.12 (a),(b),(c), respectively).

In the long wavelength limit $(|q| \ll 1)$, the perturbation growth rate is given by

$$
\omega= \pm i q \Lambda^{1 / 2}+O\left(q^{2}\right)
$$

where

$$
\Lambda=\gamma \theta_{2}\left(1+4 \beta \theta_{1}\right) .
$$

Inspecting Eq. (55), the quantity $\Lambda$ is seen to be positive, thus it leads to exponential in $t$ perturbation growth. Furthermore, it remarkably agrees with the asymptotically computed $\Omega$ values given in Eq. (28). Table 1 shows typical values for $\Omega^{1 / 2}, \Lambda^{1 / 2}$ as well as the numerically generated slopes found from system (11) for different soliton propagation constants $\mu$.

The situation for negative $\beta$ proves to be much different than what we have so far encountered. This is most evident from the fact that $q_{1}$ can now be negative for some $\beta$. In this case, the formation of an otherwise nonexistent finite band of linearly stable modes followed by a measurable interval of unstable Fourier wavenumbers occurs. This can be explained by noticing that for $q^{2}$ less than $-q_{1}>0$ the right-hand side of Eq. (52) is positive, hence $\omega \in \mathbb{R}$. Further increasing $q$ i.e. when $q^{2}$ lies in the interval $\left(-q_{1}, q_{2}^{2}\right)$ the right hand side of Eq. 52 switches sign, thus $\omega$ becomes purely complex. For fixed $\beta$, Fig. A.8 shows that there exists some soliton eigenvalue $\nu$ for which the ratio $\theta_{1}=E_{2} / E_{1}$ becomes large enough for all $|\mu|>|\nu|$ to force $q_{1}$ to switch sign and, as a result, the interval $\left(0,-q_{1}\right)$ shrinks to zero. Thus the soliton becomes unstable for perturbations with small wavenumbers. The transition from a positive to a negative $q_{1}$ value occurs when $\theta_{1}<-1 /(4 \beta)$ and is accompanied by the formation of a finite-size band of linearly stable modes $(\Lambda<0$ for $\beta, \gamma<0)$. Interestingly enough, $\Omega$ becomes negative as well when $0<\theta_{1}<-1 /(4 \beta)$, a surprising agreement with the asymptotic result discussed in Sec. 4 given the fact that we used a simple form for the variational ansatz. Finally, two typical examples corresponding to negative and positive $q_{1}$ values are shown in Fig. A.12(d-e), respectively, for parameters $\beta=-1 / 10$ and $\gamma=-1$. Overall, we observe a good agreement between the numerically computed and semi-analytically obtained stable/unstable perturbation eigenvalues.

\section{Direct numerical simulations}

In this section we perform several direct numerical simulations to confirm the linear stability results obtained in Secs. 34 and 5 as well as report on the development of nonlinear instability. We have numerically solved the Cauchy problem associated with Eq. (1) using a fourthorder split-step integration method in $t$ and Fourier spectral discretization in $x, y$ with boundary conditions: $\phi$ decays rapidly to zero as $|x| \rightarrow \infty$. The initial condition used in the simulations is

$$
\phi(x, y, 0)=\psi(x)+\varepsilon\left[\partial_{x} \psi \cos (Q y)+i \psi \sin (Q y)\right],
$$

where $|\varepsilon| \ll 1$ and $Q$ is the linear transverse perturbation wavenumber that corresponds to either a linearly stable or unstable mode. This type of perturbation requires periodic boundary conditions in the $y$-direction and allows one to focus on and excite specific perturbations with prescribed wavenumber $Q$ depicted in Fig. A.5 or Fig. A.6, rather than exciting the entire unstable spectrum (by integrating Eq. (10) over all wavenumbers $q$ with their corresponding amplitudes $f_{q}$ and $g_{q}$ which are localized in $x$ and $q$ ) which would then resemble a noisy perturbation.

First consider the positive $\beta$ case. The dynamic evolution of a moderate amplitude soliton under the combined effects of a periodically modulated perturbation in the transverse $y$-direction and longitudinal localization is shown in the top row of Fig. A.13 On a relatively short time scale, an exponentially growing neck-type instability develops (consistent with the linear theory - see Fig. A.5(a)) and ultimately leads to a full break up of the mode into a sequence of localized bright spots. The distance between adjacent filaments is approximately 4.19 units, which is roughly the period $(2 \pi / Q)$ of the transverse perturbation. To validate the linear stability results 
presented in Secs. 3 and 5 we repeat the numerical experiments, this time with wavenumber $Q>q_{\text {cut }}$ corresponding to $\operatorname{Im} \omega(Q)=0$. As one can see from the bottom row in Fig. A.13 for short times (linear stability regime) the wave pattern remains almost undisturbed compared to the initial state and develops weak bounded oscillations in the $y$-direction.

Next we shift our focus to the negative dispersion/diffraction case $(\beta<0)$. Here we study nonlinear dynamics of transverse perturbations superimposed on top of a soliton stripe. The propagation constant $\mu$ is chosen such that the linear theory (asymptotic and variational) presented in Secs. 4 and 5 predicts the existence of three linear stable or unstable Fourier bands, which happens when $q_{1}<0$. With this at hand, we have simulated Eq. (1) with initial condition (56) for various values of $Q$ residing in each linear stability/instability band. When $Q$ falls into the first stable linear band $\left(0<Q^{2}<-q_{1}\right)$, full numerical simulations reveal that the soliton almost preserves its initial shape on the order of short time scale $1 / \operatorname{Im} \omega_{\max }(q) \approx 0.28$ (see Fig. A.14 top row). This observation seems to persist well beyond that time scale. On the other hand, for the same fixed $q_{1}$, but with $Q^{2}$ chosen now inside the interval $\left(-q_{1}, q_{2}^{2}\right)$ (corresponding to linearly unstable modes) the soliton experiences severe instability and eventually disintegrates into an array of well separated two-dimensional bright spots as shown in the bottom row of Fig. A.14. Perturbations with wavenumbers $Q^{2}$ belonging to the third linear stable band $\left(q_{2}^{2}, \infty\right)$ were also examined and found to remain bounded up to $t=1$, conferring with linear theory.

As a final remark, we have performed an extensive numerical experiment regarding the long time behavior of the two-dimensional filaments, in particular, the question of collapse. Figure A.15 shows the maximum magnitude $\|\phi(t)\|_{\max }=\max _{x, y}|\phi(x, y, t)|$ as a function of $t$ for different values of $\beta$ corresponding to initial condition (56) with fixed power $\|\psi\|_{2}^{2}=2$. The perturbation wavenumber $Q$ corresponds to a linearly unstable eigenmode. As one can see from Fig. A.15, the max norm of the solution remains bounded and oscillates for long time scales.

\section{Conclusions}

In this paper we have studied the dynamics and formation of coherent structures that result from the development of instabilities for families of one-dimensional localized waves due to the presence of transverse perturbation. Our model equation is the two-dimensional nonlinear Schrödinger equation in the presence of a fourth-order dispersion/diffraction. The linear stability analysis predicts the existence of a finite band of unstable Fourier modes for which small transverse perturbations grow exponentially in $t$ and lead to the break up of the soliton stripe. On time scales longer than the inverse of the growth rate Im $\omega_{\max }$, full nonlinear simulations reveal the formation of arrays of periodic two-dimensional filaments. The numerical linear stability analysis is supported by analytical results based on perturbation theory and variational Lagrangian model reduction.

\section{Acknowledgements}

The work of J.T.C. and Z.H.M was supported in part by NSF grant number DMS-0908599.

\section{Appendix A. Spectral renormalization method}

In this section we outline the spectral renormalization method [59] used to numerically construct soliton solutions. Other numerical methods based on functional optimization of Sobolev gradients 68] can also be implemented. Solutions to Eq. (3) are sought in the form $\phi(x, y, t)=r u(x) e^{i \mu t}$ where $r$ is a yet to be determined renormalization factor and $u(x)$ is the real-valued mode profile satisfying

$$
\frac{1}{2} \partial_{x}^{2} u-\beta \partial_{x}^{4} u+\gamma r^{2} u^{3}-\mu u=0
$$

Multiplying Eq. A.1 by $u$ and integrating over the whole space gives

$$
r^{2}=\frac{\frac{1}{2}\left\|\partial_{x} u\right\|_{2}^{2}+\beta\left\|\partial_{x}^{2} u\right\|_{2}^{2}+\mu\|u\|_{2}^{2}}{\gamma\|u\|_{4}^{4}} .
$$

The function $u(x)$ is thus obtained by the following fixedpoint iteration scheme

$$
\hat{u}_{n+1}=\frac{\{\zeta-\mu\} \hat{u}_{n}+\gamma r_{n}^{2} \mathcal{F}\left[u_{n}^{3}\right]}{\frac{1}{2} k^{2}+\beta k^{4}+\zeta},
$$

where $r_{n}^{2}=\left.r^{2}\right|_{u=u}$ and $\mathcal{F}, \mathcal{F}^{-1}$ are the forward and inverse Fourier transforms respectively defined by

$$
\hat{f}=\mathcal{F}[f]=\frac{1}{\sqrt{2 \pi}} \int_{\mathbb{R}} f(x) e^{-i k x} d x,
$$

and

$$
f=\mathcal{F}^{-1}[\hat{f}]=\frac{1}{\sqrt{2 \pi}} \int_{\mathbb{R}} \hat{f}(k) e^{i k x} d k .
$$

In iteration scheme A.3 the parameter $\zeta$ is chosen such that $\zeta=\mu$ for $\beta>0$ (in which case $\mu$ is also positive) and $\zeta<0$ satsifying $1<16 \beta \zeta$ when $\beta$ is negative. Alternatively, one may instead fix the $L^{2}(\mathbb{R})$ norm of $\psi$ i.e. $\|\psi\|_{2}^{2}=N$. In this case, the fixed-point spectral renormalization algorithm reads

$$
\begin{gathered}
r_{n}^{2}=\frac{N}{\left\|u_{n}\right\|_{2}^{2}}, \\
\mu_{n}=\frac{\gamma r_{n}^{2}\left\|u_{n}\right\|_{4}^{4}-\frac{1}{2}\left\|\partial_{x} u_{n}\right\|_{2}^{2}-\beta\left\|\partial_{x}^{2} u_{n}\right\|_{2}^{2}}{\left\|u_{n}\right\|_{2}^{2}},
\end{gathered}
$$




$$
\hat{u}_{n+1}=\frac{\left\{\zeta-\mu_{n}\right\} \hat{u}_{n}+\gamma r_{n}^{2} \mathcal{F}\left[u_{n}^{3}\right]}{\frac{1}{2} k^{2}+\beta k^{4}+\zeta},
$$

for values of $\zeta$ discussed above, until convergence is reached.

\section{References}

[1] A. Chen, M. Segev and D.N. Christodoulides, Optical spatial solitons: historical overview and recent advances, Rep. Prog. Phys., 75 (2012), 086401.

[2] Y.S. Kivshar and G.P. Agrawal, Optical solitons, Academic Press, (2003).

[3] M. Segev, Optical spatial solitons, Opt. Quant. Electron., 30 (1998), pp. 503-533.

[4] D.N. Christodoulides, F. Lederer and Y. Silberberg, Discretizing light behaviour in linear and nonlinear waveguide lattices, Nature, 424 (2003), pp. 817-823.

[5] F. Lederer, G.I. Stegeman, D.N. Christodoulides, G. Assanto, M. Segev and Y. Silberberg, Discrete solitons in optics, Phys. Rep., 463 (2008), pp. 1-126.

[6] M.J. Ablowitz, Nonlinear dispersive waves: asymptotic analysis and solitons, Cambridge University Press, (2011).

[7] E.A. Kuznetsov, A.M. Rubenchik and V.E. Zakharov, Soliton stability in plasmas and hydrodynamics, Phys. Rep., 142 (1986), pp. 103-165.

[8] P.G. Kevrekidis, D.J. Frantzesakakis and R. Carretero-Gonzalez, Emergent Nonlinear Phenomena in Bose-Einstein Condensates, Springer (2008).

[9] A.L. Hodgkin and A.F. Huxley, A quantitative description of membrane current and its application to conduction and excitation in nerve, J. Physiol. 117 (1952), pp. 500-544.

[10] A.S. Davydov and N.I. Kislukha, Solitary excitons in onedimensional molecular chains, Physica Status Solidi (b), 59 (1973), pp 465-470.

[11] M.C. Cross and P.C. Hohenberg, Pattern formation outside of equilibrium, Phys. Rev. Mod. Phys., 65 (1993), pp. 851-1112.

[12] T.B. Benjamin and J.E. Feir, The disintegration of wave trains on deep water, J. Fluid Mech., 27 (1967), pp. 417-430.

[13] T. Taniuti and H. Washimi, Self-trapping and instability of hydromagnetic waves along the magnetic field in a cold plasma, Phys. Rev. Lett., 21 (1968), pp. 209-212.

[14] F.Kh. Abdullaev and J. Garnier, Modulational instability of electromagnetic waves in birefringent fibers with periodic and random dispersion, Phys. Rev. E, 60 (1999), pp. 1042-1050.

[15] C.K.R.T. Jones, R. Marangell, P.D. Miller, and R.G. Plaza, Spectral and modulational stability of periodic wavetrains for the nonlinear Klein-Gordon equation, J. Differential Equations, 257 (2014), pp. 4632-4703.

[16] K. Tai, A. Hasegawa and A. Tomita, Observation of modulational instability in optical fibers, Phys. Rev. Lett., 56 (1986), pp. 135-138.

[17] L. Salasnich, A. Parola and L. Reatto, Modulational instability and complex dynamics of confined matter-wave solitons, Phys. Rev. Lett., 91 (1986), 080405.

[18] R. Malendevich, L. Jankovic, G.I. Stegeman and J.S. Aitchison, Spatial modulation instability in a Kerr slab waveguide, Opt. Lett., 26 (2001), pp. 1879-1881.

[19] H. Fang, R. Malendevich, R. Schiek and G.I. Stegeman, Spatial modulational instability in one-dimensional lithium niobate slab waveguides, Opt. Lett., 25 (2000), pp. 1786-1788.

[20] D. Kip, M. Soljacic, M. Segev, E. Eugenieva and D.N. Christodoulides, Modulation instability and pattern formation in spatially incoherent light beams, Science, 290 (2000), pp. 495-498.

[21] J. Meier, G.I. Stegeman, D.N. Christodoulides, Y. Silberberg, R. Morandotti, H. Yang, G. Salamo, M. Sorel and J.S. Aitchison, Experimental observation of discrete modulational instability, Phys. Rev. Lett., 92 (2004), 163902
[22] K.G. Makris, Z.H. Musslimani, D.N. Christodoulides and S. Rotter, Constant-intensity waves and their modulation instability in non-Hermitian potentials, arXiv:1503.08986, (2015).

[23] Z.H. Musslimani, K.G. Makris, R. El-Ganainy and D.N. Christodoulides, Optical solitons in PT periodic potentials, Phys. Rev. Lett., 100 (2008), 030402.

[24] K.G. Makris, R. El-Ganainy, D.N. Christodoulides and Z.H. Musslimani, Beam dynamics in Pt symmetric optical lattices, Phys. Rev. Lett., 100 (2008), 103904.

[25] P.A.E.M. Janssen and J.J. Rasmussen, Nonlinear evolution of the transverse instability of plane-envelope solitons, Phys. Fluids, 26 (1983), pp. 6586-6587.

[26] Y.S. Kivshar and D.E. Pelinovsky, Self-focusing and transverse instabilities of solitary waves, Phys. Rep., 331 (2000), pp. 117195.

[27] J.D. Carter and H. Segur, Instabilities in the two-dimensional cubic nonlinear Schrödinger equation, Phys. Rev. E, 68 (2003), 045601(R).

[28] V.E. Zakharov and A.M. Rubenchik, Instability of waveguides and solitons in nonlinear media, Sov. Phys. JETP, 38 (1974), pp. 494-500.

[29] A.V. Marmaev, M. Saffman, D.Z. Anderson, and A.A. Zozulya, Propagation of light beams in anisotropic nonlinear media: From symmetry breaking to spatial turbulence, Phys. Rev. A, 54 (1996), pp. $870-879$.

[30] V. Tikhonenko, J. Christou, and B. Luther-Davies, Three dimensional bright spatial soliton collision and fusion in a saturable nonlinear medium, Phys. Rev. Lett., 76 (1996), pp. 26982701.

[31] M.A. Hoefer and B. Ilan, Dark solitons, dispersive shock waves, and transverse instabilities, Multiscale Model. Simul., 10 (2012), pp. 306-341.

[32] G. Theocharis, D.J. Frantzeskakis, P.G. Kevrekidis, B.A. Malomed, and Y.S. Kivshar, Ring Dark Solitons and Vortex Necklaces in Bose-Einstein Condensates, Phys. Rev. Lett., 90 (2003), 120403.

[33] Z.H. Musslimani and J. Yang, Transverse instability of strongly coupled dark-bright Manakov vector solitons, Opt. Lett., 26 (2001), pp. 1981-1983.

[34] C. Anastassiou, M. Soljačić, M. Segev, E.D. Eugenieva, D.N. Christodoulides, D. Kip, Z.H. Musslimani, and J.P. Torres, Eliminating the transverse instabilities of Kerr solitons, Phys. Rev. Lett., 85 (2000), pp. 4888-4891.

[35] V.A. Brazhnyi and V.M. Pérez-García, Stable multidimensional soliton stripes in two-component Bose-Einstein condensates, Chaos Solitons Fractals, 44 (2011), pp. 381-389.

[36] Z.G. Chen, M. Segev, T.H. Coskun, D.N. Christodoulides, and Y.S. Kivshar, Incoherently coupled dark-bright photorefractive solitons, Opt. Lett., 21 (1996), pp. 1821-1823.

[37] Z.G. Chen, M. Segev, T.H. Coskun, and D.N. Christodoulides, Observation of incoherently coupled photorefractive spatial soliton pairs, Opt. Lett., 21 (1996), pp. 1436-1438.

[38] Z.H. Musslimani, M. Segev, A, Nepomnyashchy, and Y.S. Kivshar, Suppression of transverse instabilities for vector solitons, Phys. Rev. E, 60 (1999), pp. R1170-R1173.

[39] Y. Kodama and M.J. Ablowitz, Transverse instability of breathers in resonant media, J. Math. Phys., 21 (1980), pp. 928931.

[40] S.-P. Gorza, B. Deconinck, Ph. Emplit. T. Trogdon, and M. Haelterman, Experimental demonstration of the oscillatory snake instability of the bright soliton of the $(2+1) D$ hyperbolic nonlinear Schrödinger equation, Phys. Rev. Lett., 106 (2011), 094101.

[41] D.E. Pelinovsky, E.A. Rouvinskaya, O.E. Kurkina, and B. Deconinck, Short-wave transverse instabilities of line solitons of the two-dimensional hyperbolic nonlinear Schrödinger equation, Theoret. Math. Phys., 179 (2014), pp. 452-461.

[42] G. Fibich and B. Ilan, Optical light bullets in a pure Kerr medium, Opt. Lett., 29 (2004), pp. 887-889.

[43] D.N. Christodoulides and R.I. Joseph, Femtosecond solitary waves in optical fibers-beyond the slowly varying approximation, 
Appl. Phys. Lett., 47 (1985), pp. 76-78.

[44] A. Calini and C.M. Schober, Dynamical criteria for rogue waves in nonlinear Schrödinger models, Nonlinearity, 25 (2012), pp. 99116.

[45] C. Conti, Quantum gravity simulation by nonparaxial nonlinear optics, Phys. Rev. A, 89 (2014), 061801(R).

[46] A.B. Cavalcanti, J.C. Cruz, H.R. da Cruz and A.S. GouveiaNeto, Modulation instability in the region of minimum groupvelocity dispersion of single-mode optical fibers via an extended nonlinear Schrödinger equation, Phys. Rev. A, 43 (1991), pp. 6162-6165.

[47] S. Pitois and G. Millot, Experimental observation of a new modulational instability spectral window induced by fourth-order dispersion in a normally dispersive single-mode optical fiber, Opt. Comm., 226 (2003), pp. 415-422.

[48] K. Kitayama, K. Okamoto and H. Yoshinaga, Extended fourphoton mixing approach to modulational instability, J. Appl. Phys., 64 (1988), pp. 1279-1287.

49] F.Kh. Abdullaev, S.A. Darmanyan, S. Bischoff, P.L. Christiansen and M.P. Sorensen, Modulational instability in optical fibers near the zero dispersion point, Opt. Comm., 108 (1994), pp. 60-64.

[50] W. Hong, Modulational instability of optical waves in the high dispersive cubic-quintic nonlinear Schrödinger equation, Opt. Comm., 213 (2002), pp. 172-182.

[51] A.G. Shagalov, Modulational instability of nonlinear waves in the range of zero disperison, Phys. Lett. A, 239 (1998), pp. 41-45.

[52] C.J. Pethick and H. Smith, Bose-Einstein condensates in dilute gases, Cambridge University Press, (2001).

[53] M.J. Ablowitz and H. Segur, Solitons and the inverse scattering transform, SIAM Press, (1981).

[54] J. Lega, J.V. Moloney and A.C. Newell, Swift-Hohenberg equation for lasers, Phys. Rev. Lett., 73 (1994), pp. 2978-2981.

[55] E. DelRe, F. Di Mei, J. Parravicini, G. Parravicini, A. J. Agranat, and C. Conti, Subwavelength anti-diffracting beams propagating over more than 1,000 Rayleigh lengths, Nature Photontics, 9 (2015), pp. 228-232.

[56] N. Bulso and C. Conti, Effective dissipation and nonlocality induced by nonparaxiality, Phys. Rev. A, 89 (2014), 023804.

[57] B. Crosignani, P. Di Porto, and A. Yariv, Nonparaxial equation for linear and nonlinear optical propagation, Opt. Lett., 22 (1997), pp. 778-780.

[58] J.T. Cole and Z.H. Musslimani, Band gaps and lattice solitons for the higher-order nonlinear Schrödinger equation with a periodic potential, Phys. Rev. A, 90 (2014), 013815.

[59] M.J. Ablowitz and Z.H. Musslimani, Spectral renormalization method for computing self-localized solutions to nonlinear systems, Opt. Lett., 30 (2005), pp. 2140-2142.

[60] M. Karlsson and A. Höök, Soliton-like pulses governed by fourth order dispersion in optical fibers, Opt. Comm., 104 (1994), pp. 303-307.

[61] V.I. Karpman, Influence of high-order dispersion on selffocusing. I. Qualitative investigation, Phys. Lett. A, 160 (1991), pp. 531-537. V.I. Karpman and A.G. Shagalov Influence of highorder dispersion on self-focusing. II. Numerical investigation, Phys. Lett. A, 160 (1991), pp. 538-540.

[62] G. Fibich, B. Ilan, and G. Papanicolaou, Self-focuing with fourth-order dispersion, SIAM J. Appl. Math., 62 (2002), pp. 1437-1462.

[63] L.N. Trefethen, Spectral Methods in MATLAB, SIAM, Philadelphia (2000).

[64] B.A. Malomed, Soliton management in periodic systems, Springer, (2006).

[65] B.A. Malomed, Variational methods in nonlinear fiber optics and related fields, Progress in Optics, 43 (2002), pp. 71-193.

[66] V.M. Pérez-García, H. Michinel, J.I. Cirac, M. Lewenstein and P. Zoller, Dynamics of Bose-Einstein condensates: Variational solutions of the Gross-Pitaevskii equations, Phys. Rev. A, 56 (1997), pp. 1424-1432.

[67] D. Anderson, A. Bondeson and M. Lisak, Transverse instability of soliton solutions to nonlinear Schrödinger equations, J.
Plasma Phys., 21 (1979), pp. 259-266.

[68] J.J. Garcia-Ripoll and V.M. Pérez-García, Optimizing Schrödinger functionals using Sobolev gradients: applications to quantum mechanics and nonlinear optics, SIAM J. Sci. Comput., 23 (2001), pp. 1316-1334.
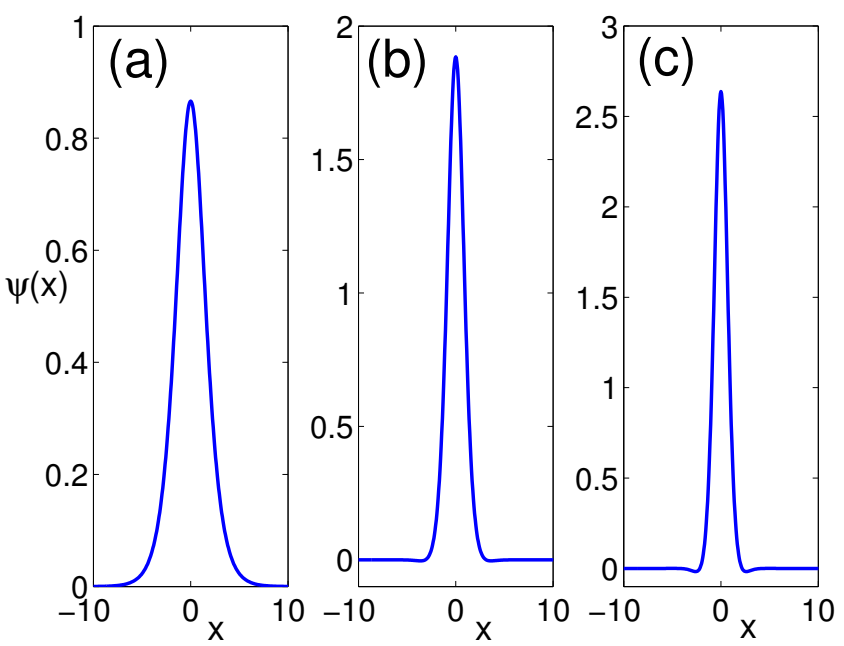

Figure A.1: Line soliton solutions to Eq. 3 for $\beta=0.1, \gamma=+1$ and soliton eigenvalues (a) $\mu=0.4$, (b) $\mu=2$ and (c) $\mu=4$.
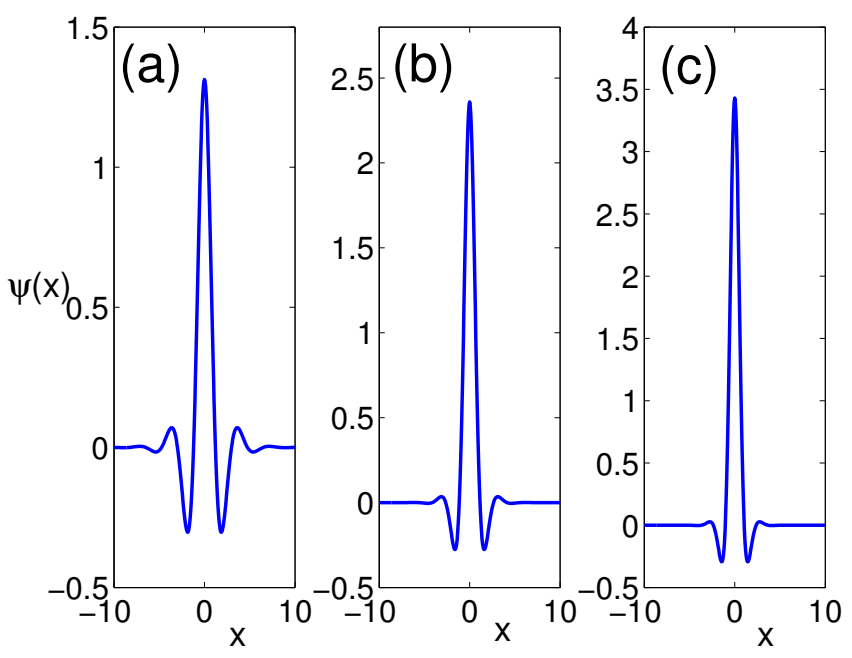

Figure A.2: Same as in Fig. A.1 but for parameters $\beta=-0.1, \gamma=$ -1 and soliton eigenvalues (a) $\mu=-1.5$, (b) $\mu=-4$ and (c) $\mu=-8$. 
(a)
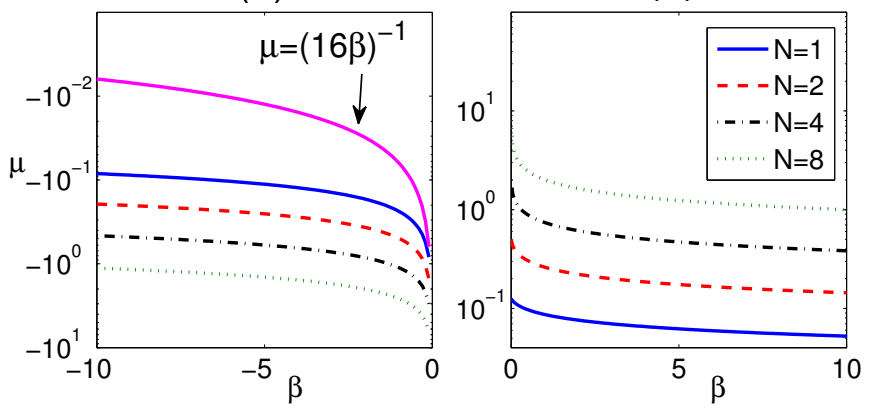

Figure A.3: Soliton existence curves with fixed transverse power $\|\psi\|_{2}^{2}=N$ for (a) negative and (b) positive $\beta, \gamma$ values. The curve labels in panel (a) are the same as in (b).
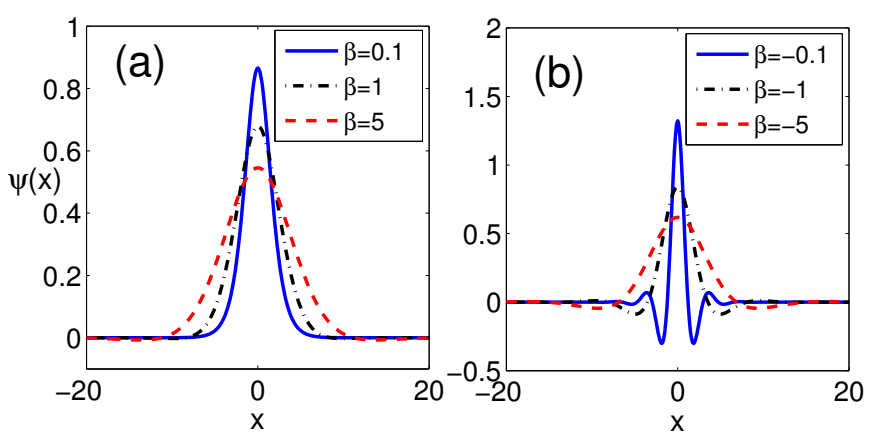

Figure A.4: Large $\beta$ soliton solutions to Eq. 3 with fixed transverse power $\|\psi\|_{2}^{2}=2$ for (a) positive and (b) negative $\beta, \gamma$ values.
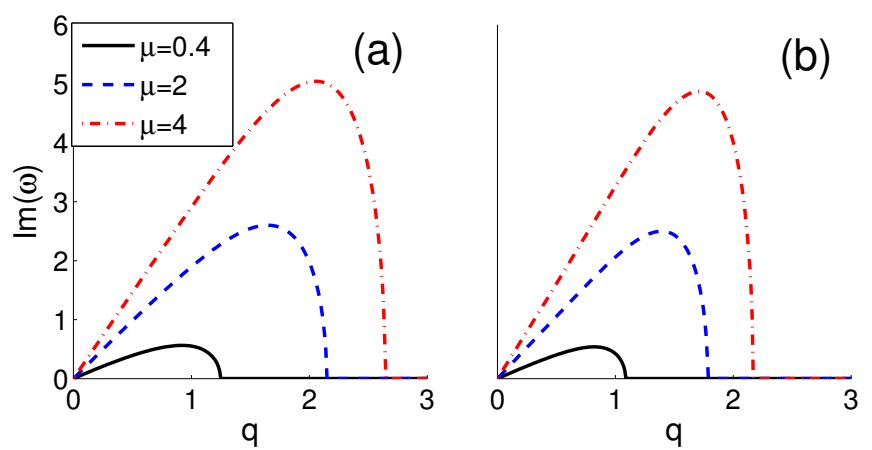

Figure A.5: The imaginary part of the spectrum numerically generated by solving eigenvalue problem 11 with $\gamma=+1$ for (a) $\beta=0.1$ and (b) $\beta=0.25$ and several eigenvalues $\mu$. The solid, dashed, and dashed-dotted curves in panel (a) correspond to the three solutions shown in Fig. A.1.
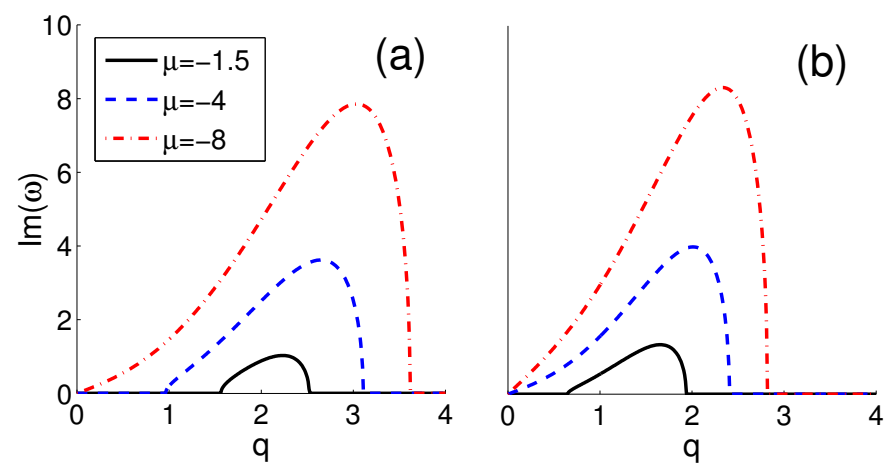

Figure A.6: The imaginary part of the spectrum numerically generated by solving eigenvalue problem 11 with $\gamma=-1$ for (a) $\beta=-0.1$ and (b) $\beta=-0.25$ and several eigenvalues $\mu$. The solid, dashed, and dashed-dotted curves in panel (a) correspond to the three solutions shown in Fig. A.2
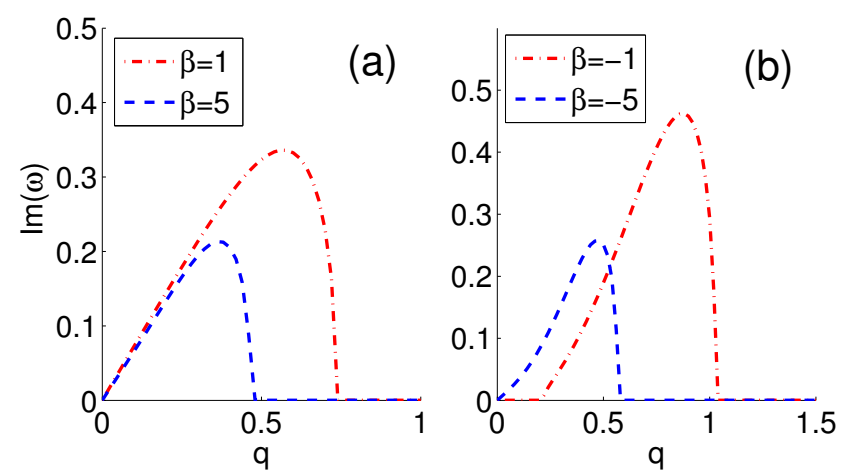

Figure A.7: The imaginary part of the spectrum numerically generated by solving eigenvalue problem with (a) $\gamma=1$ and (b) $\gamma=-1$ for a fixed transverse power $\|\psi\|_{2}^{2}=2$ and various values of $\beta$. 

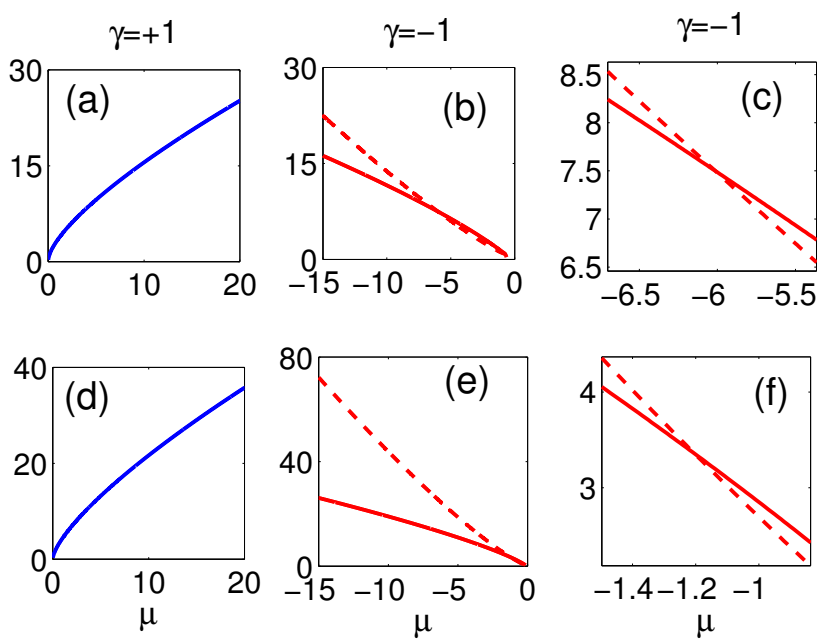

Figure A.8: Soliton power curves $\|\psi\|_{2}^{2}$ (solid line) as a function of propagation constant $\mu$ for (a) $\beta=0.1$, (d) $\beta=0.5$, (b-c) $\beta=-0.1$, and (e-f) $\beta=-0.5$. Shown also the dependence of the "kinetic energy" $4|\beta||| \partial_{x} \psi \|_{2}^{2}$ on $\mu$ (dashed line) for negative $\beta$. The third column is a zoom-in of the middle column around the intersection point.
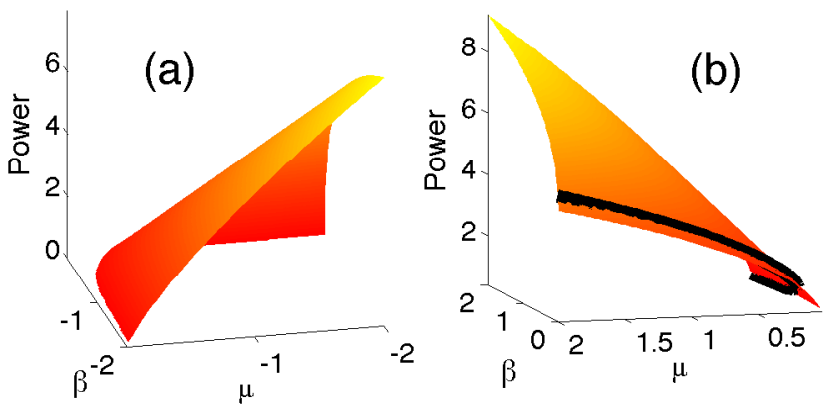

Figure A.9: Transverse power surfaces $\left(\|\psi\|_{2}^{2}\right)$ as a function of $\mu$ and $\beta$ for (a) $\gamma=-1$ and (b) $\gamma=+1$. In panel (b), the one-dimensional solid curve $\|\psi\|_{2}^{2}(\mu)=(10 \mu)^{1 / 2}, \mu=(25 \beta)^{-1}$ corresponds to the existence curve of the exact solution given in Eq. (4).
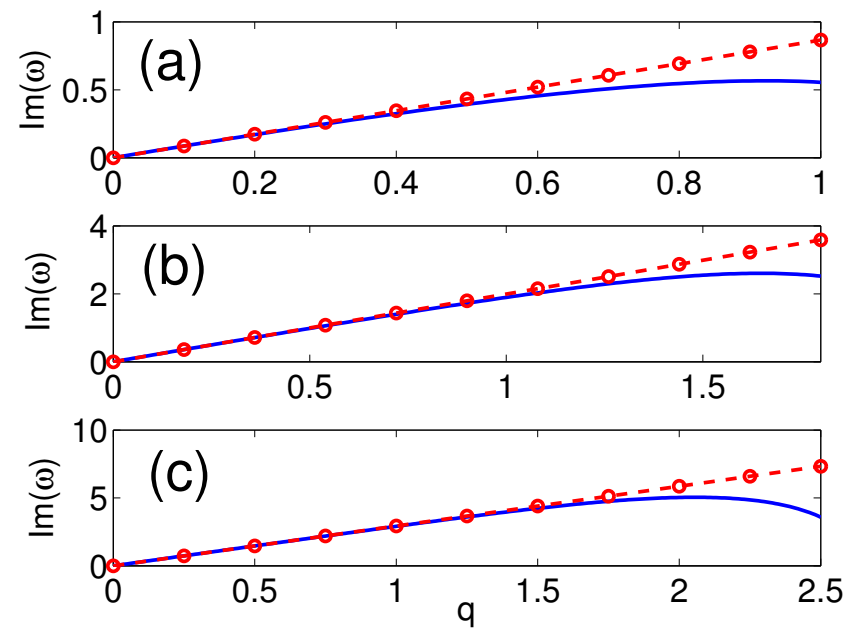

Figure A.10: The imaginary part of the TI spectrum computed from Eq. 27 (dashed line with circles) and by numerical solution of eigenvalue problem 111 (solid line) for $\beta=0.1$ and $\gamma=+1$. The panels correspond to solution eigenvalues (a) $\mu=0.4$, (b) $\mu=2$ and (c) $\mu=4$. The approximate slopes $\Omega^{1 / 2}$ are (a) 0.866 , (b) 1.992 and (c) 2.931 .

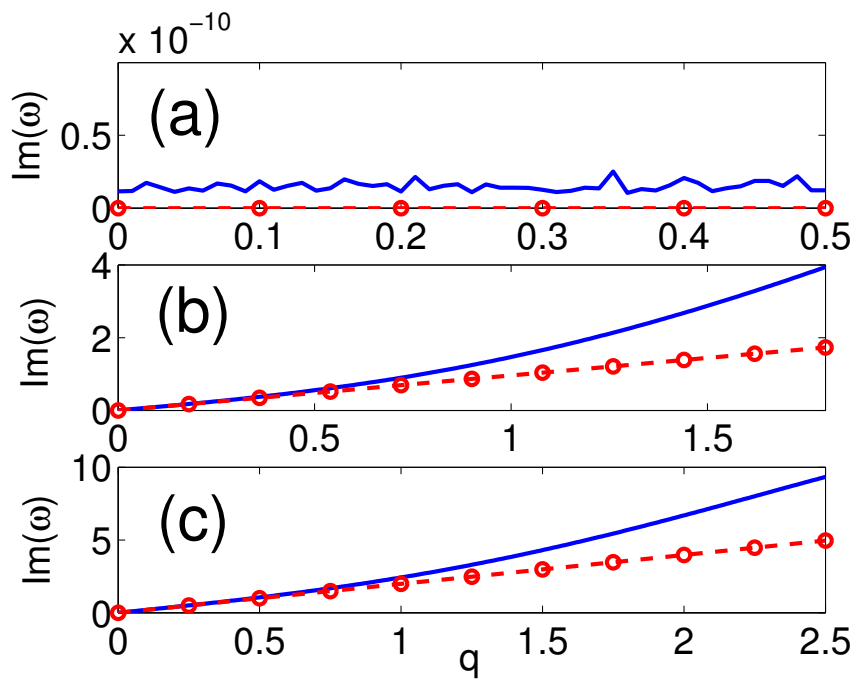

Figure A.11: Same as Fig. A.10 but for $\beta=-0.1$ and $\gamma=-1$. The panels correspond to solution eigenvalues (a) $\mu=-4$, (b) $\mu=-8$ and (c) $\mu=-12$. The approximate slopes $\Omega^{1 / 2}$ are (a) 0 , (b) 0.962 , and (c) 1.984 . 

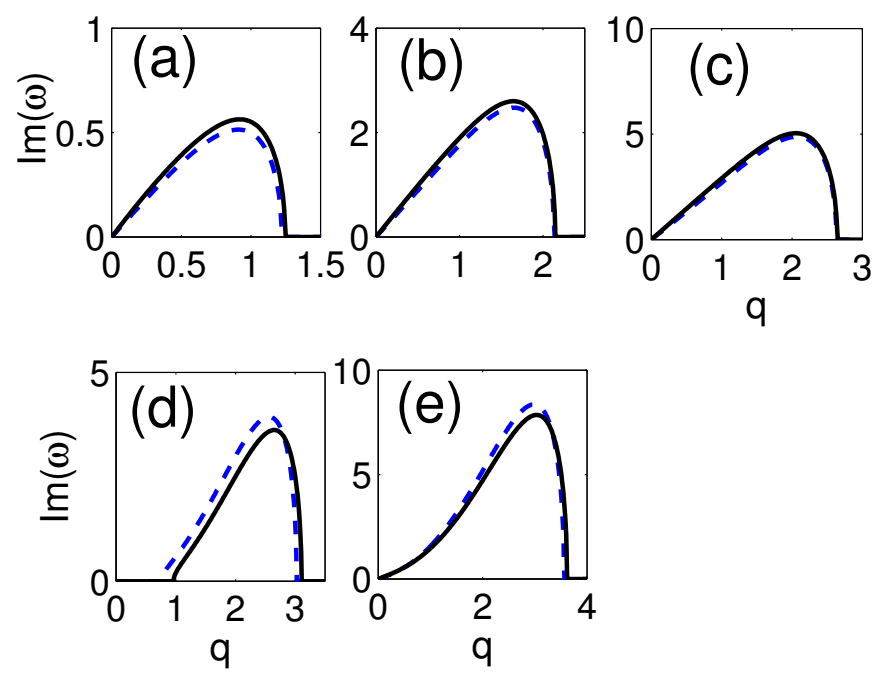

Figure A.12: Instability growth rate obtained from Eq. 52 (dashed line) for $\beta=0.1, \gamma=+1$ and eigenvalues (a) $\mu=0.4$, (b) $\mu=2$ and (c) $\mu=4$. The solid line is the numerically computed eigenvalues from system 11p. The bottom row is the same as the top row, but for parameters $\beta=-0.1, \gamma=-1$ and eigenvalues (d) $\mu=-4$ and (e) $\mu=-8$.
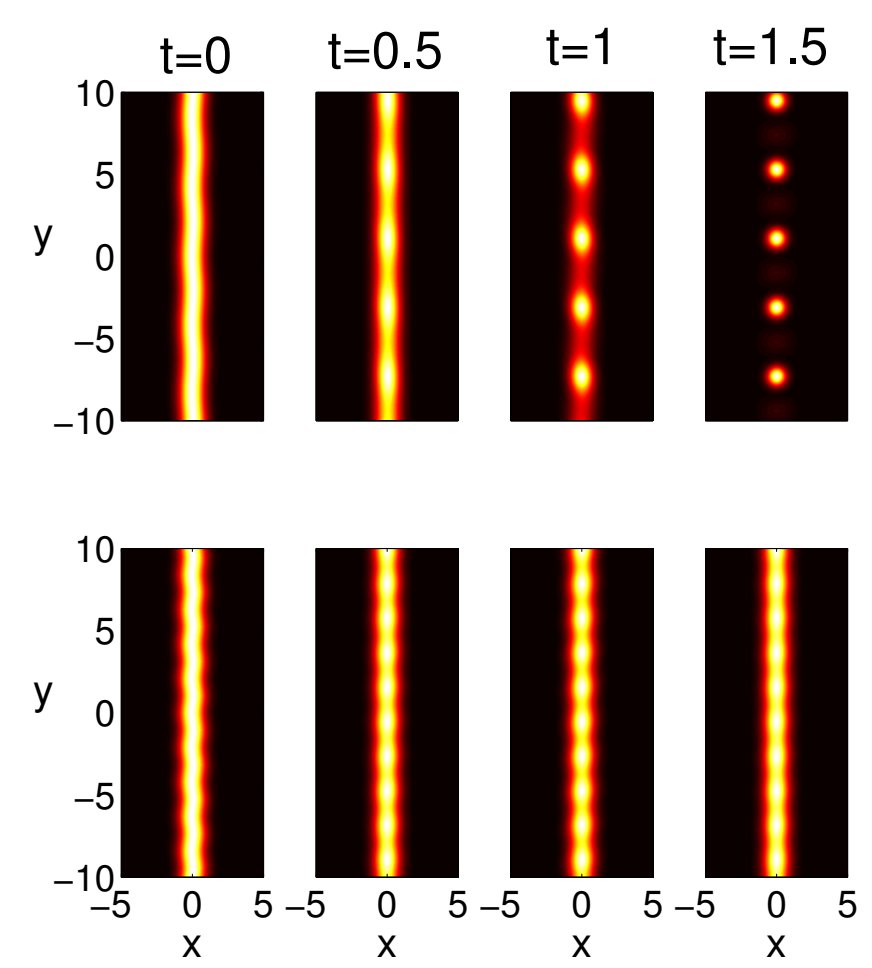

Figure A.13: A snapshot top view of the intensity field $|\phi(x, y, t)|^{2}$ at various $t$ obtained from numerical simulation of Eq. (1) using initial condition 56. The initial soliton profile $\psi(x)$ is chosen from Fig. A.1 (b) with parameters $\beta=0.1, \mu=2$ and $\gamma=+1$. The perturbation parameters are $\varepsilon=0.05$ and (top row) $Q=1.5$, (bottom row) $Q=3$.
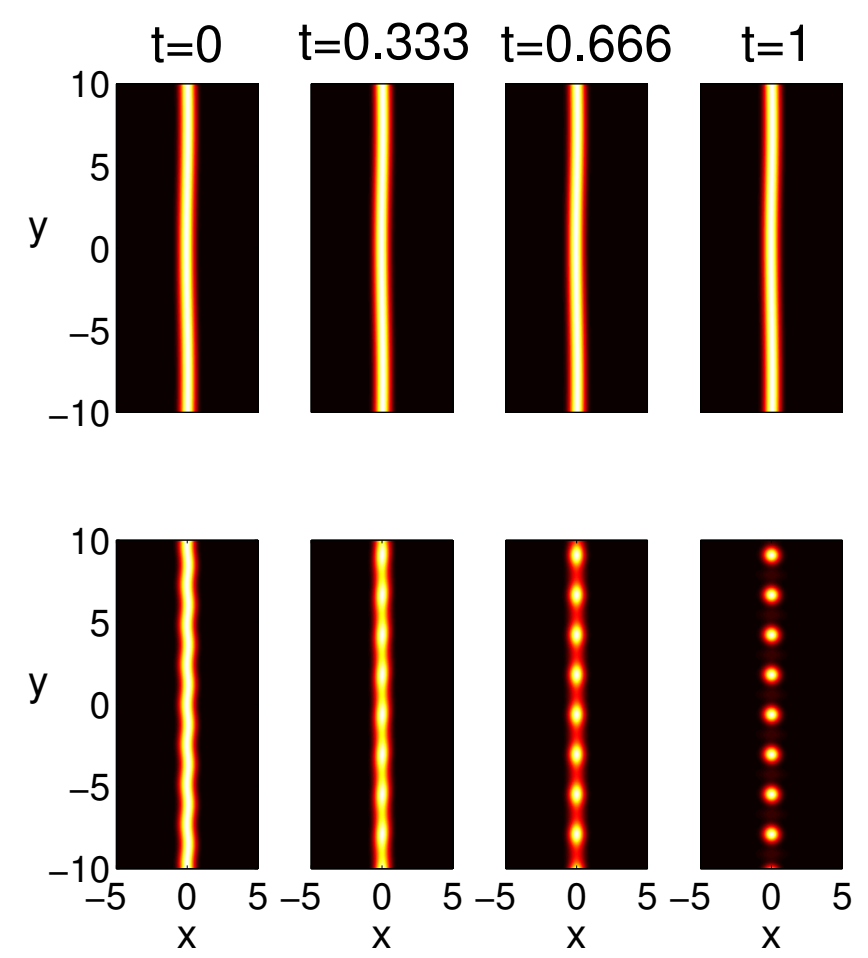

Figure A.14: A snapshot top view of the intensity field $|\phi(x, y, t)|^{2}$ at various $t$ obtained from numerical simulation of Eq. (1) using initial condition 56. The initial soliton profile $\psi(x)$ is chosen from Fig. A.2 (b) with parameters $\beta=-0.1, \mu=-4$ and $\gamma=-1$. The perturbation parameters are $\varepsilon=0.05$ and (top row) $Q=0.4$, (bottom row) $Q=2.6$

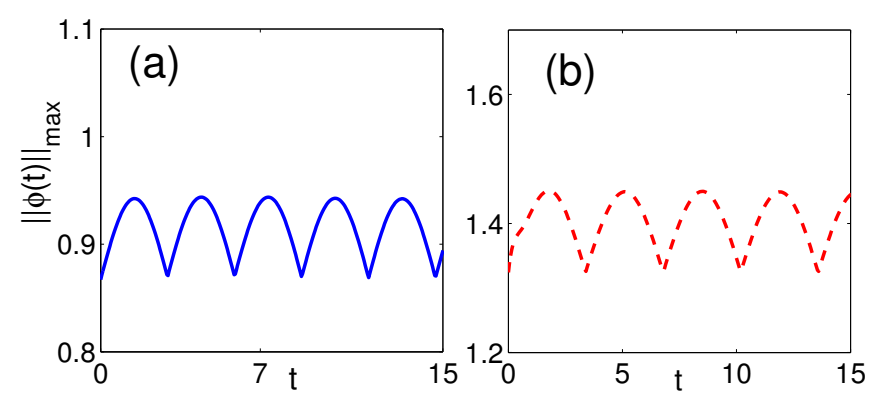

Figure A.15: Maximum solution amplitude $\|\phi(t)\|_{\max }=$ $\max _{x, y}|\phi(x, y, t)|$ for $\|\psi\|_{2}^{2}=2$ initially perturbed according to Eq. 56 for (a) $\beta=0.1, \gamma=1, Q=1.5$ and (b) $\beta=-0.1, \gamma=$ $-1, Q=2.6$. In both caes $\varepsilon=0.05$. 